\title{
Experimental study of $\beta$ spectra using Si detectors
}

\author{
Abhilasha Singh ${ }^{1,2, *}$, Xavier Mougeot ${ }^{1}$, Benoit Sabot $^{1}$, Didier Lacour ${ }^{1}$, and Abdel-Mjid Nourreddine $^{2}$ \\ ${ }^{1}$ CEA, LIST, Laboratoire National Henri Becquerel (LNE-LNHB), CEA-Saclay 91191, Gif/Yvette Cedex, France \\ ${ }^{2}$ Institut Pluridisciplinaire Hubert Curien, University of Strasbourg, CNRS-IN2P3-UMR 7178, 23 rue du Loess, BP 28, F-67037, \\ Strasbourg Cedex 2, France
}

\begin{abstract}
.
Several scientific users from different communities, such as nuclear medicine, ionizing radiation metrology, nuclear energy industries, and fundamental physics are seeking for a precise knowledge of beta spectra. Consequently, it is of great interest to investigate the spectral shape of beta decaying nuclei with best possible precision. In this work, we aim to investigate precisely $\beta^{-}$decays. For this purpose, we have developed a quasi $4 \pi$ beta spectrometer based on Silicon detectors (PIPS ${ }^{\circledR}$ ). The measurement system is characterized by conversion electron peaks of ${ }^{109} \mathrm{Cd}$ and ${ }^{207} \mathrm{Bi}$ and has been compared with Monte Carlo simulations using nuclear decay data. The preliminary measurements of ${ }^{14} \mathrm{C}$ (allowed transition) and ${ }^{36} \mathrm{Cl}$ (second forbidden non-unique transition) decays have been performed and compared with simulations using the PENELOPE Monte Carlo code.
\end{abstract}

\section{Introduction}

The study of the shape of beta spectra is expanding interest widely as it has rarely been investigated in the past 50 years. The current nuclear data is lacking with a precise knowledge of beta spectra which is required by several users like nuclear energy industry, nuclear medicine, ionizing radiation metrology, fundamental physics etc. Most of the available data are mainly on allowed and first forbidden transitions. Knowledge on transitions of higher forbiddenness orders is very limited and it is necessary to investigate precisely the shapes of beta decay, which is pivotal in several ongoing research such as dark matter studies [1] and anti-neutrino anomaly [2]. A few years ago, a theoretical code named BETASHAPE was developed at Laboratoire National Henri Becquerel (LNE-LNHB), which is the primary laboratory for ionizing radiations in France [3]. This code calculates the beta spectra for allowed and forbidden unique transitions and also includes a database of 130 experimental shape factors available in literature. The code takes into account the atomic screening effect and the radiative corrections [4]. The calculations are more complicated in the case of forbidden nonunique transitions because of their high sensitivity to nuclear structure. As each forbidden non-unique transition would require a detailed study and an input from a precise nuclear model, BETASHAPE treats the transition as an allowed or a forbidden unique one of same variation of total angular momentum, the so-called $\xi$ approximation. Therefore, experimental studies on the shape of beta spectra with the lowest possible uncertainty are highly essential to constrain and validate the theoretical predictions.

*e-mail: abhilasha.singh@cea.fr, abhilasha.13111993@gmail.com
At LNE-LNHB, we have developed a new beta spectrometer in a quasi $4 \pi$ geometry with two Si detectors for a precise measurement of spectra with maximum endpoint energy of $850 \mathrm{keV}$, detailed in section 2.1. This spectrometer with a close $4 \pi$ geometry allows to reduce the effect of back-scattering, which distorts beta spectrum shape significantly, by summing the energy deposited in each detector for every emitted particle. Studies were performed to quantify the spectrum distortion due to the quality of radioactive source and special care has been taken for the radioactive source preparation technique which is detailed in section 2.2. The characterization of the device has been done using conversion electron peaks of ${ }^{109} \mathrm{Cd}$ and ${ }^{207} \mathrm{Bi}$ and preliminary beta spectra measurements have been performed for ${ }^{14} \mathrm{C}$ and ${ }^{36} \mathrm{Cl}$ and are explained in section 3 .

\section{Experimental Set-up}

\subsection{Measurement system}

The difficulty in precise beta spectra measurement arises from the measurement system itself. Therefore, it is very important to take into account all the sources of distortion, i.e. the detection geometry (solid angle), the detector dead layer and (back-)scattering of particles. We have designed a set-up in a quasi $4 \pi$ geometry in order to minimize the escape of particles and maximize the absorption of the emitted particle energy in coincidence mode. This configuration includes two identical Passivated Implanted Planar Silicon (PIPS ${ }^{\circledR}$ ) detectors fully depleted, each of $1 \mathrm{~mm}$ thickness and $300 \mathrm{~mm}^{2}$ surface area. In the active volume of $1 \mathrm{~mm}$ thick Si detector, electrons of energy up to $850 \mathrm{keV}$ can have full energy deposition. For a very precise measurement especially in the case of beta particles, 
it is preferable to have a detector with the thinnest possible entrance window in order to minimize the energy loss within the entrance window of detector. The ion implantation technique has the great advantage of providing a maximum thickness of $50 \mathrm{~nm}$ equivalent silicon for the PIPS ${ }^{\circledR}$ dead layer. The detectors are mounted in a detector holder in copper manufactured on purpose, which includes a dedicated design to place the radioactive source and the two preamplifiers. The device is designed in such a way that the silicon surface of each detector is maintained at distance of $3 \mathrm{~mm}$ from each other with the radioactive source in the middle as shown in figure 1 .

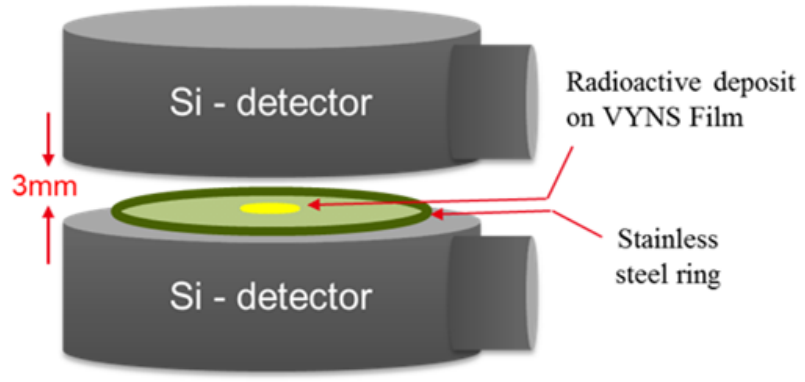

Figure 1. The basic scheme of the measurement system with two PIPS $^{\circledR}$ detectors $\left(1 \mathrm{~mm}\right.$ thickness and $300 \mathrm{~mm}^{2}$ surface area) and the radioactive source in the middle.

The detectors are connected to their respective preamplifier with a $3 \mathrm{~cm}$ long coaxial cable. This configuration reduces the capacitance and increases the signal-to-noise ratio. The whole detector assembly then goes inside a vacuum chamber, an aluminum cube of edge $17 \mathrm{~cm}$, and is fixed on a cold copper finger which comes from a liquid nitrogen Dewar [5] in order to cool down the device. Electrical connections have been arranged on purpose using an electronic feed-through. In order to get the sum of energy deposited in the two detectors, a passive module has been developed in-house. In this module, the signal from each preamplifier is given as an input and the difference of the preamplifier gain is adjusted using the sharp peak of ${ }^{207} \mathrm{Bi}$ at $975 \mathrm{keV}$. The resulting signal, i.e the sum of the two signals is then acquired using the labZY module which is a numerical acquisition module based on FGPA technology [6].

\subsection{Radioactive source}

The quality of the measured spectrum strongly depends on the quality of the radioactive source because the beta particles may lose their energy within the source material, a usual self-absorption phenomenon. This phenomenon was studied using Monte Carlo simulations by varying the thickness of the source carrier film from $0.5 \mu \mathrm{m}$ to $18 \mu \mathrm{m}$ and a significant energy loss was observed in thicker films especially at low energy. Consequently, we have prepared radioactive sources with $0.5 \mu \mathrm{m}$ VYNS film and $0.7 \mu \mathrm{m}$ Mylar ${ }^{\circledR}$ film as source support. The VYNS film is a good candidate for our purpose but it is very fragile and the radioactive source prepared on it can not be preserved more than 3 months. Therefore, we are now using a newly available Mylar ${ }^{\circledR}$ film of $0.7 \mu$ m thickness, which is more adaptive than VYNS film. We have studied the energy losses in both films using Monte Carlo simulations and the difference is negligible. For both, the source preparation technique is the same and follows mainly 4 steps:

- The preparation of source support by placing film on a stainless steel ring;

- The deposition of latex microspheres by electrospraying, which serve as a seeding agent and increase the wettability of the film;

- Deposition of the radioactive drop;

- Drying of the source.

The film is supported on a stainless steel ring $\left(\phi_{\text {outer }}=25 \mathrm{~mm}, \phi_{\text {inner }}=15 \mathrm{~mm}\right.$, thickness $\left.=0.15 \mu \mathrm{m}\right)$. The latex microspheres that have been electrosprayed allow a homogeneous radioactive deposit $(\phi=10 \mathrm{~mm}$ and thickness $=0.15 \mu \mathrm{m}$ ). As the decay constant of signal is typically $50 \mu \mathrm{s}$, we have prepared sources with a typical activity of $1 \mathrm{kBq}$ in order to minimize the pile-up contribution. Moreover, the shaping parameters in the labZY module intrinsically accounts for pile-up rejection [6].

\section{Preliminary results}

\subsection{Energy calibration}

A precise measurement needs a definite characterization of the measurement system. For the characterization of our set-up, measurements have been carried out for ${ }^{207} \mathrm{Bi}$ and ${ }^{109} \mathrm{Cd}$. The measured spectra were analyzed using COLEGRAM [7], a spectrum analysis software developed at LNHB which fits experimental data using a non-linear least square method. The energy threshold of the measurement was observed to be $22 \mathrm{keV}$. The conversion electron peaks were fitted using Gaussian functions and the FWHM (Full Width Half Maximum) was found to be $10 \mathrm{keV}$ at $62 \mathrm{keV}$. Furthermore, the presented experimental geometry has been implemented in PENELOPE, a well-known Monte Carlo code [8]. Each component associated with the experimental geometry was precisely taken into account. Simulation has been performed for ${ }^{207} \mathrm{Bi}$ using the PENNUC module which uses the evaluated data from DDEP (Decay Data Evaluation Project) [9] to manage the cascade of particles from the primary decay generated by the atomic and nuclear relaxation processes (gamma-rays, conversion electrons, X-rays and Auger electrons). The simulated spectrum was convoluted with the energy resolution and has been compared with the measured spectrum. As shown in figure 2, an excellent agreement is observed between data and measurement.

The well defined conversion electrons peaks of ${ }^{207} \mathrm{Bi}$ and ${ }^{109} \mathrm{Cd}$ have been analyzed to determine the energy calibration curve of the measurement system. As shown in figure 3, a linear response from $22 \mathrm{keV}$ to $1065 \mathrm{keV}$ has been observed. This calibration has been applied to the further measurements of ${ }^{14} \mathrm{C}$ and ${ }^{36} \mathrm{Cl}$. 


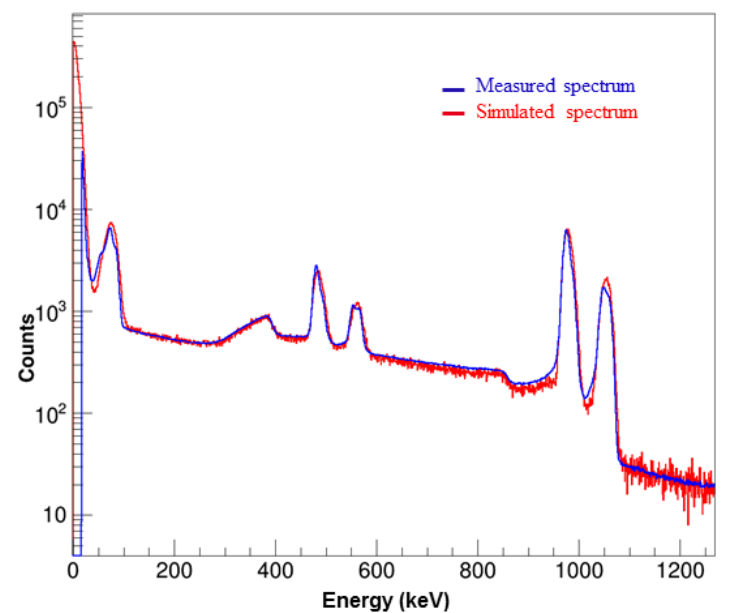

Figure 2. Comparison of measured spectrum (blue) with simulated spectrum (red) of ${ }^{207} \mathrm{Bi}$ using PENNUC module.

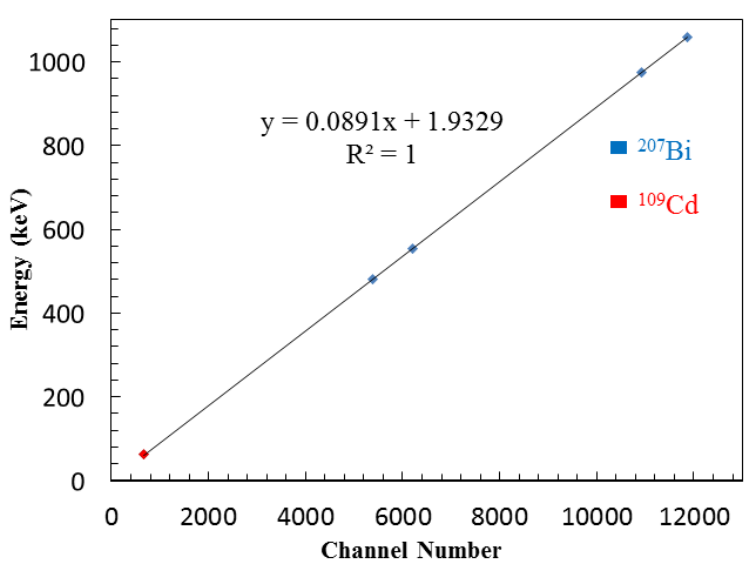

Figure 3. The energy calibration of the detectors with conversion electron peaks of ${ }^{207} \mathrm{Bi}$ and ${ }^{109} \mathrm{Cd}$.

\section{$3.2{ }^{14} \mathrm{C}$}

The first beta spectrum measurement has been performed for ${ }^{14} \mathrm{C}$ decay with a maximum endpoint energy of 156.476 (4) $\mathrm{keV}$ [10]. The radioactive source of ${ }^{14} \mathrm{C}$ was prepared on Mylar ${ }^{\circledR}$ film and the corresponding experimental geometry was simulated with PENELOPE. The ${ }^{14} \mathrm{C}$ beta decay is an allowed transition but is well-known for exhibiting a nuclear structure effect which drastically increase its half-life. Therefore, instead of a theoretical spectrum, an experimental shape factor available from Kuzminov and Osetrova [11] was used as an input of the simulation. The resulting beta spectrum was convoluted with energy resolution of $10 \mathrm{keV}$ and compared with measured spectrum. Both the spectra were normalized by integration between $40 \mathrm{keV}$ and $80 \mathrm{keV}$. As shown in figure 4 , an excellent agreement is found between the simulated and measured spectra.

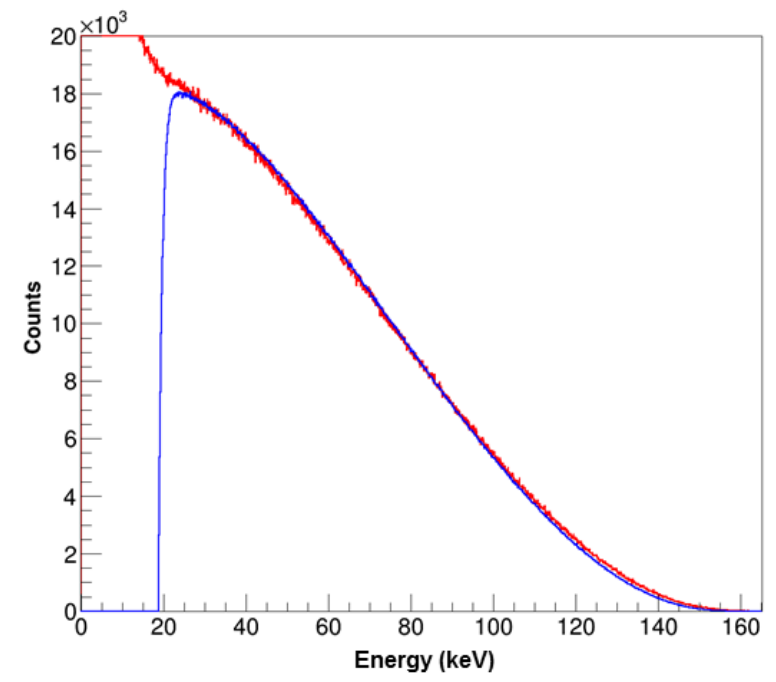

Figure 4. Comparison of measured spectrum (blue) with simulated spectrum (red) ${ }^{14} \mathrm{C}$ decay (allowed transition).

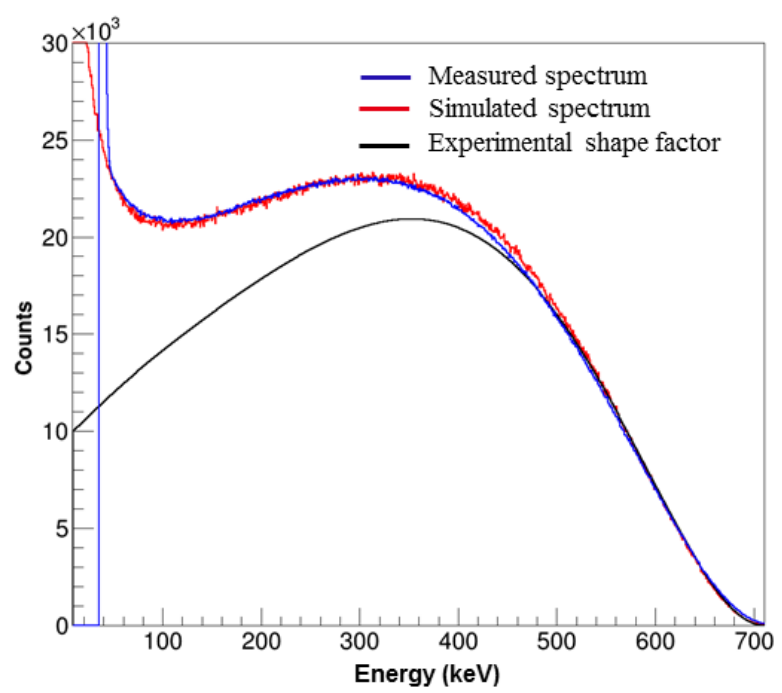

Figure 5. Comparison of measured spectrum (blue) with simulated spectrum (red). The initial spectrum (black) used as input of the simulation comes from an experimental shape factor from Rotzinger et al. [12] of ${ }^{36} \mathrm{Cl}$ decay (second forbidden non-unique transition).

\section{$3.3{ }^{36} \mathrm{Cl}$}

The second beta spectrum measurement was done for ${ }^{36} \mathrm{Cl}$ decay which is a second forbidden non-unique transition with a maximum energy of 709.53 (4) $\mathrm{keV} \mathrm{[10].} \mathrm{The}$ source of ${ }^{36} \mathrm{Cl}$ was prepared on VYNS film and the corresponding experimental geometry has been simulated in PENELOPE. As this is forbidden non-unique transition, the theoretical prediction is difficult due the sensitivity to nuclear structure. Therefore, we used as an input of the simulation a shape factor extracted from a measurement carried out by Rotzinger et al. [12] with a cryogenic detector. The simulated spectrum was convoluted with energy 
resolution and is compared with our measurement in figure 5 .

The input spectrum is also given (black curve) to highlight the distortion due to our apparatus. The spectra are normalized by integration between $200 \mathrm{keV}$ and $300 \mathrm{keV}$. The measured and simulated spectra were observed to be in good agreement.

\section{Conclusion}

Our quasi $4 \pi$ beta spectrometer is operational and we have successfully realized some preliminary measurements. Studies were performed to quantify the distortion due to the source support material and we have adapted a novel technique for radioactive source preparation using thin films. The characterization of the measurement system was validated by Monte Carlo simulations using the PENELOPE code, with the PENNUC module for the management of the decay scheme and the atomic relaxation. The preliminary measurements of ${ }^{14} \mathrm{C}$ and ${ }^{36} \mathrm{Cl}$ spectra were compared with precise PENELOPE simulations and an excellent agreement was found which clearly validated the approach of beta spectral measurement with this technique. However, it is noteworthy that the current geometrical configuration does not completely prevent the escape of particles. Therefore, some distortions still remain which need to be corrected for before any extraction of an experimental shape factor. This distortion can be corrected through a deconvolution process by implementing the response of detection system. Such an algorithm is currently under development.

\section{References}

[1] Pradler, J., et al., Phy. Lett. B. 720 399, (2013)

[2] Sonzogni, A., et al., Phys. Rev. Lett. 119 112501, (2017)

[3] Mougeot, X., EPJ Web of Conferences 146 12015, (2017)

[4] Mougeot, X., Phys. Rev. C 91 05554, (2015)

[5] Bisch, C., Nucl. Data. Sheets. 120C 95, (2014)

[6] http://www.labzy.com

[7] Ruellan, H., Nucl. Inst. Methods. A 369 95, (1996)

[8] Salvat, F., PENELOPE-2008 - A Code System for Monte Carlo Simulation of Electron and Photon Transport NEA report NEA/NSC/DOC (2009)

[9] Salvat, F., PENELOPE-2014 - A Code System for Monte Carlo Simulation of Electron and Photon Transport NEA report NEA/NSC/DOC (2015)

[10] Be, M.-M., et al.,Table of Radionuclides, volume 7 of Monographie BIPM-5., 7 (2013)

[11] Kuzminov, V.V., et al.,Physics of Atomic Nuclei 61 1292 (2000)

[12] Rotzinger, H., et al., J. Low Temp. Phys 1511087 (2008) 